\title{
Reliability and clinical usefulness of the personality inventory for DSM-5 in clinically referred adolescents: A preliminary report in a sample of Italian inpatients
}

\author{
Antonella Somma $^{\mathrm{a}}$, Andrea Fossati ${ }^{\mathrm{a}, *}$, Arianna Terrinoni $^{\mathrm{b}}$, Riccardo Williams ${ }^{\mathrm{c}}$, \\ Ignazio Ardizzone ${ }^{\mathrm{c}}$, Fiorella Fantini ${ }^{\mathrm{c}}$, Serena Borroni ${ }^{\mathrm{d}}$, Robert F. Krueger ${ }^{\mathrm{e}}$, \\ Kristian E. Markon ${ }^{\mathrm{f}}$, Mauro Ferrara ${ }^{\mathrm{b}}$ \\ ${ }^{a}$ Department of Human Studies, LUMSA University, Rome, Italy, and San Raffaele Hospital, Milan, Italy \\ ${ }^{\mathrm{b}}$ Department of Pediatrics and Child and Adolescent Neurology and Psychiatry, "Sapienza" University of Rome, Rome, Italy \\ 'Department of Dynamic and Clinical Psychology, "Sapienza" University of Rome, Rome, Italy \\ ${ }^{\mathrm{d}}$ Faculty of Psychology, Vita-Salute San Raffaele University, Milan, Italy \\ ${ }^{\mathrm{e}}$ Department of Psychology, University of Minnesota, Minneapolis, MN, U.S.A. \\ ${ }^{\mathrm{f}}$ Department of Psychology, University of Iowa, Iowa City, IA, U.S.A.
}

\begin{abstract}
Background: The DSM-5 Alternative Model of Personality Disorders (AMPD) provides the opportunity to integrate the needed developmental perspective in the assessment of personality pathology. Based on this model, Krueger and colleagues (2012) developed the Personality Inventory for DSM-5 (PID-5), which operationalizes the proposed DSM-5 traits.

Methods: Eighty-five consecutively admitted Italian adolescent inpatients were administered the Italian translation of the PID-5, in order to obtain preliminary data on PID-5 reliability and clinical usefulness in clinically referred adolescents.

Results: With the possible exception of the PID-5 Suspiciousness scale, all other PID-5 scales evidenced adequate internal consistency reliability (i.e., Cronbach's $\alpha$ values of at least .70, most being greater than .80 ). Our data seemed to yield at least partial support for the construct validity of the PID-5 scales also in clinical adolescents, at least in terms of patterns of associations with dimensionally assessed DSM-5 Section II PDs that were also included in the DSM-5 AMPD (excluding Antisocial PD because of the participants' minor age). Finally, our data suggested that the clinical usefulness of the PID-5 in adolescent inpatients may extend beyond PDs to profiling adolescents at risk for life-threatening suicide attempts. In particular, PID-5 Depressivity, Anhedonia, and Submissiveness trait scales were significantly associated with adolescents' history of life-threatening suicide attempts, even after controlling for a number of other variables, including mood disorder diagnosis.
\end{abstract}

Discussion: As a whole, our study may provide interesting, albeit preliminary data as to the clinical usefulness of PID-5 in the assessment of adolescent inpatients.

(C) 2016 Elsevier Inc. All rights reserved.

\section{Introduction}

Notwithstanding the assessment of personality pathology in adolescence is important, personality dysfunction is commonly misdiagnosed or completely missed in the adolescent population. Indeed clinically significant emotion-

* Corresponding author at: Clinical Psychology and Psychotherapy Unit, San Raffaele Turro Hospital, via Stamira d'Ancona 20, 20127, Milan, Italy. Tel.: +39 02 26433241; fax: +39 0226433408 .

E-mail address: fossati.andrea@hsr.it (A. Fossati). al dysregulation and externalizing behaviors are sometimes overlooked during this developmental period because they are mistaken for transient, age-related abnormal behaviors [1]. Adolescence is usually considered as a developmental stage characterized by impulsivity, emotional and psychological disruptions, rapid mood swings, and increased vulnerability to breakdowns in adaptive behaviors [2,3]. Adolescence is furthermore characterized as a fluid developmental period, in which dramatic changes take place [4]. Indeed, adolescence may not represent a smooth transition to adulthood for at least a significant portion of adolescents, 
and the biological and social transitions associated with adolescence may prove challenging for some adolescents and their families [4].

Personality pathology in younger age groups has traditionally been a delicate topic, with many clinicians showing a reluctance to use Axis II labels in childhood or adolescence [5] However, the dearth of research in this area has gradually given way to a growing research literature [e.g.,6,7], and there is a growing awareness that maladaptive behaviors associated with personality pathology in adulthood may be disentangled from adaptive turmoil during adolescence [4]. This is particularly true for borderline personality disorder (BPD), which has recently been suggested to be a valid and reliable diagnosis in adolescence e.g., [4,7-8]. Indeed, authors have cast doubts on the notion that BPD suddenly appears in an individual when s/he turns 18. Psychometric data clearly indicate that personality disorders (PDs), particularly BPD, can be reliably diagnosed in adolescence using descriptive diagnostic criteria [9-11]; however, a dimensional perspective may be particularly important for conceptualizing PD pathology among youth because it is better able to account for the developmental fluctuations and increased heterogeneity that have been reported in younger samples [e.g.,9]. The availability of a dimensional model of PD pathology and of reliable and valid measures for assessing PD dimensions in adolescence seems to be vital for implementing early intervention programs [e.g., 7,8,10] which may promote more adaptive developmental pathways, averting many of the negative outcomes of PDs in adulthood.

Despite these advances, there remains a serious dearth of studies that examine the psychometric properties of measures to assess maladaptive personality functioning in youth e.g., [e.g.,12]. Early identification and treatment of PDs is dependent on the careful and accurate assessment of personality pathology in adolescents. Valid and reliable instruments may helpfully complement clinical assessment. Such instruments should take into account the recent emphasis on dimensional models of maladaptive personality functioning [13], especially in youth, as categorical approaches to personality assessment focus narrowly on specific symptoms, and do not allow for the study of the entire range of personality pathology dysfunction [e.g.,6].

To address various concerns with the personality disorder (PD) categories in use since the third edition of Diagnostic and Statistical Manual of Mental Disorders DSM-III [14]; - for example, lack of empirically validated cutoffs, extensive comorbidity, and temporal instability [15] — an Alternative Model of Personality Disorder (AMPD) was provided in DSM-5 Section III (American Psychiatric Association, 2013a), along with traditional PD symptom criteria listed in $D S M-5$ Section II (which reprints $D S M-I V[16]$ axis II PD symptom criteria). A core component of the DSM-5 AMPD is an empirically based model of maladaptive personality domain and traits [17] which represents a synthesis of existing dimensional models of personality pathology, focused on maladaptive variants [e.g.,18-21].

In particular, the AMPD allows clinicians to assess five major domains of maladaptive personality - namely, negative affectivity (frequent and intense experiences of high levels of a wide range of negative emotions), detachment (avoidance of socio-emotional experience), antagonism (behaviors that put the individual at odds with other people), disinhibition (orientation toward immediate gratification and impulsive behavior), and psychoticism (a wide range of culturally incongruent odd, eccentric, or unusual behaviors and cognition). These domains also contain 25 specific maladaptive personality trait facets, or specific aspects of the domains (e.g., anxiousness, depressivity, attention seeking, risk taking, eccentricity, etc. [17]). Based on this model, Krueger and colleagues [22] developed the Personality Inventory for DSM-5 (PID-5); the PID-5 is a 220-item self-report questionnaire with a 4-point response scale; it yields 25 primary scales that can be combined to also delineate 5 higher order scales (negative affectivity, detachment, antagonism, disinhibition, and psychoticism). Indeed, a burgeoning literature is yielding consistent evidence for the reliability and validity of the PID-5 in community dwelling and clinical adults [17]. The PID-5 has been translated and validated in a number of languages, including Italian [23]. In the present study, we relied on the Italian translation of the PID-5 in order to assess DSM-5 AMPD traits and domains.

Up to now only De Clercq and colleagues [5] administered the PID-5 to a sample of 434 community dwelling adolescents; results indicated an acceptable reliability for the majority of the PID-5 facets and a tendency toward structural convergence of the adolescent PID-5 factor structure with the adult proposal, although the higher-order structure differed slightly from the adult structure, perhaps, due to the interference of normative developmental processes throughout childhood, that may influence the indicative value of specific traits across time. De Clercq and colleagues [5] strongly stressed the need for further research on the validity of the PID-5 in younger age groups. To our knowledge, no study on the psychometric properties of the PID-5 in clinically referred adolescents has been carried out.

Previous research also indicates that personality pathology among adolescents may be associated with significant impairment in this age group. For instance, BPD features in adolescents have been related to clinically significant behaviors, such as suicidal thoughts and behaviors [e.g.,24]. Given the high prevalence of self-harm/suicide-related behaviors in the general adolescent population (mean lifetime prevalence of suicide-related behaviors $=16.1 \%$; [25]), and the fact that suicide-related behaviors may predict future PDs, life-threatening suicidal attempts have important implications for the conceptualization and treatment of PDs during early stages of the disorder, when targeted treatment is most effective [26].

Starting from these considerations, we aimed at obtaining preliminary evidence of internal consistency reliability and 
clinical usefulness of the PID-5 scales in a sample of Italian adolescent inpatients. In order to evaluate the clinical usefulness of the PID-5 scales, we tested two major hypotheses. First, we evaluated if the expected PID-5 trait profiles for five (namely, Avoidant PD, Obsessive-Compulsive PD, Schizotypal PD, Narcissistic PD, and Borderline PD) of the six PDs that are listed in DSM-5 Section III were empirically reproduced in our sample in terms of associations between PID-5 traits scale scores and dimensional scores (i.e., number of criteria) of $D S M-5$ Section II PDs based on semi-structured interviews. (Of note, participants' minor age prevented us from assessing DSM-5 Section II Antisocial PD, as well as its PID-5 profile.) Second, we evaluated the associations between PID-5 scales and participants' history of life-threatening suicidal attempts.

\section{Method}

\subsection{Participants}

The sample was composed of 85 adolescent inpatients who were consecutively admitted to the Child Psychiatry Department and the Department of Dynamic and Clinical Psychology of the "Sapienza" University of Rome, Italy, from October 2014 to July 2015. The adolescent unit of the department is highly specialized in the assessment and stabilization of adolescents who showed severe personality problems. Institutional review board approval was obtained. All participants were admitted on a voluntary basis after obtaining written informed consent to admission by each adolescent and his/her parents. Sixty six (77.6\%) participants were female, whereas 19 (22.4\%) participants were male; participants' mean age was 15.31 years, $S D=1.49$ years (range 12-17 years). All participants were attending school when the study was carried out; $75(88.2 \%)$ adolescents were attending high school, whereas 10 (11.8\%) adolescents were attending junior high school. Potential participants were screened for the following exclusionary criteria: (1) IQ less than 80 (i.e. IQ was formally assessed if cognitive impairment or deterioration was suspected); (2) diagnosis of autism spectrum disorder (and other neurodevelopmental disorders), schizophrenia, schizoaffective disorder, schizophreniform disorder, or delusional disorder according to DSM-IV diagnostic criteria; (3) diagnosis of organic mental disorder according to DSM-IV diagnostic criteria. All participants in the current research passed this screen.

In our sample, all participants met criteria for at least one $D S M-I V$ Axis I diagnosis; in particular, 50 (58.8\%) adolescents met criteria for at least one $D S M-I V$ mood disorder diagnosis, $21(24.7 \%)$ adolescents received a $D S M-I V$ anxiety disorder diagnosis, $11(12.9 \%)$ adolescents were diagnosed as suffering from $D S M-I V$ other specified eating disorders, and 27 (31.8\%) adolescents met criteria for a $D S M-I V$ impulse control disorder diagnosis. $D S M-I V$ axis I diagnoses were clinically assessed by the clinicians who were following adolescents in treatment blind to scores on all measures that were used in the present study, as well as blind to the aim of this study. Independent from DSM-IV axis I diagnosis, 24 (28.2\%) adolescents reported a history of one or more life-threatening suicide attempts before admission.

All participants voluntarily took part in the study and gave their written consent to participate in the study after it had been explained to them; since all adolescent participants were of minor age, their parents provided consent for them to participate in the study. All participants were treated in accordance with the Ethical Principles of Psychologists and Code of Conduct; none of the participants received an incentive, either directly or indirectly for participating, and were administered all measures as part of their routine clinical assessment.

\subsection{Measures}

All participants were administered the Italian translation of the PID-5 [18] and the Structured Clinical Interview for Axis-II DSM-IV Personality Disorders, version 2.0 (SCID-II [27]). Although the SCID II was developed to asses DSM-IV axis II PDs, we considered it as a measure of DSM-5 Section II PDs because the PD diagnostic criteria which are listed in the DSM-5 Section II were retained with no changes from the DSM-IV axis II [28].

In the translation process, the authors closely followed Denissen, Geenen, van Aken, Gosling, and Potter's [29] indications. First, the PID-5 was translated into Italian by one of the authors (A.F.) after obtaining official permission for this translation from the American Psychiatric Association. The guiding principle was to respect the original meaning of the items; then, one of the coauthors (S.B.) reviewed the translation independently. After reaching a consensus, a native English professional translator translated the Italian version back into English. When the latest version differed from the English original, the first author, the second author and the professional translator come to an agreement on the definitive Italian translation. Then, the consensus translation was sent to the authors (R.F. K and K.E. M) for their comments; this process was iteratively pursued until final approval of the official Italian translation of the PID-5 by all authors. A similar procedure was previously followed for the Italian translation of the SCID-II [30].

All participants were administered the SCID-II blind to PID-5 scores; moreover, researchers who scored the PID-5 were blind to SCID-II assessment results, and interviewers administering SCID-II were blind to PID-5 profiles. In the present study, SCID-II was administered by trained clinical psychologists and child psychiatrists. Participants with psychiatric disorder diagnoses completed the PID-5, and were administered the SCID-II by expert trained raters after acute symptom remission according to the judgment of the clinicians who were following them in treatment (to avoid confounding effects of axis I disorders on personality disorder diagnoses [31]).

The internal consistency reliability coefficient (i.e., Cronbach's alpha) values for the PID-5-scales are listed in Table 1. 
Table 1

Cronbach's $\alpha$ and mean inter-item correlation values, descriptive statistics, gender comparisons, and correlations with age for personality inventory for DSM-5 Trait and Domain Scales $(\mathrm{N}=85)$.

\begin{tabular}{|c|c|c|c|c|c|c|c|c|c|}
\hline \multirow[b]{2}{*}{ PID-5 Trait Scales } & \multirow[b]{2}{*}{$\alpha$} & \multicolumn{3}{|c|}{ Whole sample $(N=85)$} & \multicolumn{2}{|c|}{$\begin{array}{l}\text { Male participants } \\
\qquad(n=19)\end{array}$} & \multicolumn{2}{|c|}{$\begin{array}{l}\text { Female participants } \\
\qquad(n=66)\end{array}$} & \multirow[b]{2}{*}{$r_{r-b}$} \\
\hline & & $\mathrm{MIC}$ & $M$ & $S D$ & $M$ & $S D$ & $M$ & $S D$ & \\
\hline Anxiousness & .81 & .32 & 1.89 & 0.69 & 1.49 & 0.76 & 2.01 & 0.62 & $-.41 * *$ \\
\hline Emotional lability & .89 & .55 & 2.06 & 0.72 & 1.52 & 0.78 & 2.21 & 0.63 & $-.52 * * *$ \\
\hline Hostility & .84 & .34 & 1.55 & 0.72 & 1.14 & 0.57 & 1.67 & 0.71 & $-.45 * *$ \\
\hline Perseveration & .74 & .24 & 1.40 & 0.57 & 1.09 & 0.52 & 1.49 & 0.55 & $-.39 * *$ \\
\hline Restricted affectivity & .73 & .27 & 1.12 & 0.63 & 0.89 & 0.63 & 1.18 & 0.61 & $-.32 *$ \\
\hline Separation insecurity & .82 & .40 & 1.55 & 0.74 & 0.99 & 0.52 & 1.71 & 0.71 & $-.59 * * *$ \\
\hline Submissiveness & .91 & .73 & 1.19 & 0.91 & 0.95 & 0.69 & 1.27 & 0.96 & -.17 \\
\hline Anhedonia & .87 & .46 & 1.53 & 0.73 & 1.15 & 0.69 & 1.64 & 0.71 & $-.38 *$ \\
\hline Depressivity & .96 & .66 & 1.73 & 0.90 & 1.04 & 0.77 & 1.93 & 0.83 & $-.56^{* * *}$ \\
\hline Intimacy avoidance & .89 & .56 & 0.97 & 0.81 & 0.58 & 0.63 & 1.08 & 0.83 & $-.39 * *$ \\
\hline Suspiciousness & .67 & .22 & 1.64 & 0.59 & 1.40 & 0.59 & 1.71 & 0.57 & $-.30 *$ \\
\hline Withdrawal & .92 & .54 & 1.14 & 0.76 & 0.85 & 0.62 & 1.22 & 0.78 & -.27 \\
\hline Attention seeking & .91 & .56 & 0.99 & 0.75 & 1.10 & 0.79 & 0.96 & 0.74 & .11 \\
\hline Callousness & .89 & .37 & 0.56 & 0.50 & 0.47 & 0.43 & 0.59 & 0.52 & -.16 \\
\hline Deceitfulness & .85 & .37 & 0.74 & 0.55 & 0.71 & 0.63 & 0.74 & 0.52 & -.07 \\
\hline Grandiosity & .85 & .48 & 0.45 & 0.53 & 0.69 & 0.70 & 0.38 & 0.46 & .27 \\
\hline Manipulativeness & .83 & .50 & 0.65 & 0.64 & 0.69 & 0.50 & 0.64 & 0.68 & .14 \\
\hline Distractibility & .90 & .50 & 1.62 & 0.74 & 1.53 & 0.63 & 1.65 & 0.77 & -.11 \\
\hline Impulsivity & .93 & .68 & 1.61 & 0.87 & 0.94 & 0.68 & 1.80 & 0.83 & $-.56 * * *$ \\
\hline Rigid perfectionism & .82 & .32 & 1.10 & 0.61 & 0.92 & 0.48 & 1.15 & 0.63 & -.18 \\
\hline Risk taking & .91 & .43 & 1.19 & 0.67 & 1.03 & 0.73 & 1.24 & 0.65 & -.22 \\
\hline Irresponsibility & .76 & .31 & 1.63 & 0.68 & 1.29 & 0.67 & 1.72 & 0.66 & $-.39 * *$ \\
\hline Eccentricity & .95 & .60 & 1.54 & 0.82 & 1.20 & 0.77 & 1.64 & 0.81 & $-.30 *$ \\
\hline Perceptual dysreg. & .90 & .43 & 1.18 & 0.70 & 0.79 & 0.57 & 1.29 & 0.69 & $-.41 * *$ \\
\hline Unusual beliefs & .86 & .44 & 0.98 & 0.75 & 0.83 & 0.69 & 1.02 & 0.76 & -.14 \\
\hline \multicolumn{10}{|l|}{ PID-5 Domain scales } \\
\hline Negative affectivity & .91 & .31 & 1.83 & 0.59 & 1.33 & 0.54 & 1.98 & 0.53 & $-.63 * * *$ \\
\hline Detachment & .95 & .43 & 1.21 & 0.66 & 0.86 & 0.53 & 1.32 & 0.66 & $-.39 * *$ \\
\hline Antagonism & .91 & .33 & 0.61 & 0.45 & 0.70 & 0.48 & 0.58 & 0.44 & .16 \\
\hline Disinhibition & .92 & .34 & 1.44 & 0.58 & 1.13 & 0.37 & 1.53 & 0.60 & $-.44 * *$ \\
\hline Psychoticism & .96 & .43 & 1.24 & 0.67 & 0.94 & 0.61 & 1.32 & 0.66 & $-.32 *$ \\
\hline
\end{tabular}

PID-5: Personality Inventory for DSM-5; $\alpha$ : Cronbach's alpha coefficient; MIC: mean inter-item correlation; $r_{r-b}$ : rank biserial $r$ coefficient. Statistical significance for $r_{r-b}$ coefficients was based on Mann-Whitney $U$-tests.

$* p<.05$.

$* * p<.01$.

$* * * p<.001$.

\subsubsection{Personality Inventory for DSM-5 (PID-5 [22])}

The PID-5 is a 220 -item questionnaire with a 4-point response scale $(0=$ very false or often false to $3=$ very true or often true), which was explicitly designed to measure the DSM-5 Alternative PD Model traits. Seventeen (approximately $8 \%$ ) of 220 items are reverse coded. PID-5 items are summed to compose PID-5 trait scale scores; then, PID-5 trait scales are summed to generate PID-5 domain scale scores. Each PID-5 item is scored on only one PID-5 trait scale; similarly, each PID-5 trait scale is scored on only one PID-5 domain scale. The PID-5 has 25 primary scales that load onto 5 higher order dimensions [22], and this structure is replicable [17].

Since no consistent data on the PID-5 domain structure in adolescent samples are currently available, in the present study, PID-5 domain scores were computed based on the American Psychiatric Association guidelines [32]; only 3
PID-5 trait scale scores should be average to compute PID-5 Negative Affectivity (i.e. emotional lability, anxiousness, separation insecurity), detachment (i.e. withdrawal, anhedonia, intimacy avoidance), antagonism (i.e. manipulativeness, deceitfulness, grandiosity), disinhibition (i.e. irresponsibility, impulsivity, distractibility), and psychoticism (i.e. unusual beliefs and experiences, eccentricity, perceptual dysregulation) domain scale scores.

The reliability and construct validity of the Italian translation of the PID-5 in nonclinical adult participants have been recently published [23]. In the present study, the PDs Section III total score were calculated by computing the average of the trait scores included in the PDs diagnostic criteria (e.g., the BPD Section III total score in these analyses was calculated by computing the average of the seven PID-5 trait scores included in the BPD diagnostic criteria). The reason for using the average of the traits is to include all 
possible manifestations of the PDs construct as measured by Section III in one dimensional index. This method is standard practice in previous research on adult samples (e.g. [33]).

\subsubsection{Structured Clinical Interview for DSM-IV Axis II} personality disorders, version 2.0 (SCID-II; [27])

The SCID-II is a 140-item semi-structured interview designed to provide both a categorical and dimensional (i.e., number of symptoms) assessment of DSM-IV PDs. The SCID-II was preceded by administration of its self-report screening questionnaire (PQ). The validity of the PQ as a measure for screening PD psychopathology has been previously reported [34], and SCID-II enables direct probing of negative PQ answers when this is considered clinically relevant [27]. Only the SCID-II scores for the 10 PDs that were retained in the DSM-5 were considered in the present study. The inter-rater reliability and internal consistency of the Italian translation of the SCID-II in clinical adult participants were assessed in a previous study [30].

In the present study, the inter-rater reliability (IRR) of SCID-II dimensional (i.e., number of criteria) PD diagnoses could be assessed on the first 12 consecutively admitted adolescent inpatients using a pairwise interview designs; intraclass $r$ based on one-way random effect ANOVA model was used to quantitatively assess IRR of SCID-II dimensional PD diagnoses that were considered in the present study. In this sub-sample, IRR data could not be computed for dimensionally assessed Schizotypal PD because Schizotypal PD features were almost never rated on the SCID-II by either rater 1 (median number of SCID-II Schizotypal PD features $=0.00, S D=0.89$ ) or rater 2 (median number of SCID-II Schizotypal PD features $=0.00, S D=0.90$ ). In our study, IRR was acceptable or adequate for dimensionally assessed SCID-II Avoidant PD, Narcissistic PD, and Borderline PD diagnoses, as it was indicated by interclass $r$ values of $.58, .95$, and .95 , all $p \mathrm{~s}<.05$, respectively; rather, only fair rater agreement was observed for dimensionally assessed SCID-II Obsessive Compulsive PD, intraclass $r=.43$.

\subsection{Data analyses}

Cronbach's $\alpha$ coefficient and mean inter-item correlation (MIC) were used to assess the internal consistency of the PID-5 scales. Considering the size of our sample, we preferred relying on non-parametric tests. Spearman $r$ was used to evaluate the associations between PID-5 scales and dimensionally assessed SCID-II PDs, as well as between pairs of continuous measures. Mann-Whitney $U$ test was used to evaluate the associations between adolescents' history of life-threatening suicide attempts and PID-5 scales; rank biserial $r$ coefficient [35] was used as an effect size measure for Mann-Whitney $U$ test. In order to test the effect of participants' mood disorder diagnosis on the association between life-threatening suicide history and PID-5 scales, we performed two-way ANOVAs. In particular, we relied on
Aligned Rank Transform (ART [36]); ART represents a nonparametric approach to factorial ANOVA that corrects for violations of the homogeneity of variance assumption, providing accurate nonparametric treatment for both main and interaction effects while retaining the familiarity and interpretability of the familiar parametric $F$ test [36-39]. ART relies on a preprocessing step that first "aligns" the data for each effect (main or interaction) before assigning ranks, averaged in the case of ties. Data alignment is an established process in statistics [39] by which effects are estimated as marginal means and then "stripped" from the response variable so that all effects but one are removed. After this preprocessing step, data were analyzed using a full factorial $2 \times 2$ ANOVA design (for a detailed description, see [36]), using the same procedure that was described above for parametric analyses.

Although a number of procedures have been proposed to carry out nonparametric ANCOVAs, up to now no agreed upon procedure has been suggested [37]. In order to test the effect of dimensionally assessed (i.e., number of symptoms) DSM-5 Section II BPD on the relationship between history of life-threatening suicide attempts and PID-5 scale scores, we first regressed on SCID-II BPD scale scores each PID-5 scale that showed a significant bivariate association with adolescents' history of life-threatening suicide attempts, saving raw residual scores. Then, for each participant we added to the PID-5 scale residual score the corresponding scale mean value in order to obtain the PID-5 scale score adjusted for the effect of dimensionally assessed BPD. Finally, we re-performed Mann-Whitney $U$ test on these adjusted PID-5 scores which were obtained holding constant BPD symptom effect.

ART preprocessing was carried out using the ARTool [36] computer program; all statistical analyses were carried out using the R computer program [40].

\section{Results}

\subsection{Descriptive statistics}

Cronbach's $\alpha$ and MIC values, descriptive statistics, and gender comparisons for PID-5 trait and domain scales are listed in Table 1. According to SCID-II, 80 (94.1\%) adolescent inpatients received at least one DSM-5 Section II PD diagnosis; the average number of SCID-II PD diagnoses was $1.95, S D=1.09$, whereas $53(62.3 \%)$ adolescent inpatients received two or more PD diagnoses. Based on SCID-II assessment, adolescent inpatients met on average 22.17 DSM-5 Section II PD criteria, $S D=7.04$. Borderline PD $(n=45,52.9 \%)$, Avoidant PD $(n=24$, $28.2 \%)$, Not Otherwise Specified (Mixed) PD $(n=12$, $14.1 \%)$, and Narcissistic PD $(n=10,11.8 \%)$ diagnosis were the most frequently observed SCID-II PD diagnoses in our sample. According to SCID-II, in our adolescent sample the base rate estimate for both Obsessive-Compulsive PD or Schizotypal PD diagnoses was 7.1\% $(n=6)$. 
Table 2

Dimensionally assessed borderline, narcissistic, avoidant, obsessive-compulsive, and schizotypal personality disorders: descriptive statistics and rank order correlations (Spearman r) with Personality Inventory for DSM-5 Trait Scales $(\mathrm{N}=85)$.

\begin{tabular}{|c|c|c|c|c|c|}
\hline \multirow[b]{2}{*}{ Personality Inventory for $D S M-5$ Trait Scales } & \multicolumn{5}{|c|}{ Spearman $r$ coefficients } \\
\hline & BPD & NPD & APD & OCPD & SzPD \\
\hline Anxiousness (BPD, APD) & .16 & -.04 & $.23^{*}$ & -.12 & .17 \\
\hline Emotional lability (BPD) & $.39 * * *$ & .14 & .13 & -.07 & .08 \\
\hline Hostility (BPD) & $.37 * * *$ & .14 & .00 & -.07 & -.04 \\
\hline Perseveration (OCPD) & .18 & .05 & .08 & -.06 & .02 \\
\hline Restricted affectivity (OCPD, SzPD) & -.02 & .04 & .15 & .01 & .06 \\
\hline Separation insecurity (BPD) & $.32 * *$ & .00 & .12 & -.16 & -.14 \\
\hline Submissiveness & -.02 & -.01 & .02 & -.03 & -.07 \\
\hline Anhedonia (APD) & .16 & .02 & $.31^{* *}$ & -.07 & .21 \\
\hline Depressivity (BPD) & $.40 * * *$ & -.16 & .17 & $-.23 *$ & .06 \\
\hline Intimacy avoidance (APD, OCPD) & .12 & -.01 & $.25^{*}$ & .01 & .14 \\
\hline Suspiciousness (SzPD) & $.29 * *$ & -.04 & .11 & -.16 & $.24^{*}$ \\
\hline Withdrawal (APD, SzPD) & .03 & .11 & $40^{* * *}$ & .01 & $.23 *$ \\
\hline Attention seeking (NPD) & .03 & $.29 * *$ & -.19 & .06 & -.07 \\
\hline Callousness & .11 & .12 & -.01 & .01 & .08 \\
\hline Deceitfulness & -.06 & .08 & -.19 & -.02 & -.16 \\
\hline Grandiosity (NPD) & -.05 & $.28^{* *}$ & -.16 & -.02 & .16 \\
\hline Manipulativeness & .05 & $.24 *$ & $-.23 *$ & .02 & -.12 \\
\hline Distractibility & .12 & .07 & .20 & -.16 & .15 \\
\hline Impulsivity (BPD) & $.43 * * *$ & .04 & -.04 & $-.24 *$ & -.18 \\
\hline Rigid perfectionism (OCPD) & $.26^{*}$ & $.24^{*}$ & -.02 & $.24 *$ & -.03 \\
\hline Risk taking (BPD) & $.33 * *$ & -.01 & -.16 & $-.24 *$ & -.08 \\
\hline Irresponsibility & .19 & -.07 & .05 & $-.22 *$ & -.02 \\
\hline Eccentricity (SzPD) & .11 & -.01 & .00 & -.14 & .18 \\
\hline Perceptual dysregulation (SzPD) & $.27^{*}$ & -.01 & .06 & $-.25^{*}$ & .14 \\
\hline Unusual beliefs (SzPD) & .18 & -.09 & -.10 & $-.29^{* *}$ & .19 \\
\hline$M$ (number of criteria met according SCID-II) & 4.27 & 1.87 & 2.34 & 1.00 & 1.19 \\
\hline$S D$ (number of criteria met according SCID-II) & 2.30 & 2.03 & 1.81 & 1.19 & 1.62 \\
\hline
\end{tabular}

PD: personality disorder; BPD: borderline PD; NPD: narcissistic PD; APD: avoidant PD; OCPD: obsessive-compulsive PD; SzPD: schizotypal PD; SCID-II: Structured Clinical Interview for DSM-IV Axis II Personality Disorders, version 2.0. For each PID-5 trait scale expected associations based on the DSM-5 AMPD profile for each specific PD are listed between brackets.

$* p<.05$.

** $p<.01$.

$* * * p<.001$.

\subsection{Relationships between dimensionally assessed SCID-II PD diagnoses and PID-5 scales}

In our study, the number of SCID-II PD diagnoses correlated significantly with PID-5 Negative Affectivity, Spearman $r=.24, p<.05$, and Detachment, Spearman $r=$ $.31, p<.01$, domain scales. Considering SCID-II PDs that are listed also in DSM-5 AMPD (i.e., Borderline PD, Narcissistic PD, Avoidant PD, Obsessive-Compulsive PD, and Schizotypal PD), descriptive statistics for dimensional assessment (i.e., number of criteria) and bivariate rank-order correlations with PID-5 trait scales are listed in Table 2. Since all participants were of minor age, Antisocial PD could not be formally assessed using SCID-II; thus, it could not be included in Table 2. For each PID-5 trait scale, expected associations based on the DSM-5 AMPD profile for each specific PD are listed between brackets.

When we considered the associations between each Section III PD total scores and its corresponding Section II PD, Spearman's $r$ coefficient values were $.49, .38$, and .36 , all $p \mathrm{~s}<.001$, for Borderline PD, Narcissistic PD, and Avoidant PD, respectively. The Spearman's $r$ coefficient value for the association between Section III Schizotypal PD total score and Section II Schizotypal PD was .23, $p<.05$, whereas Section III Obsessive-Compulsive PD total score did not showed a significant association with Section II Obsessive-Compulsive PD $(r=11, p>.05)$.

\subsection{Relationships between history of life-threatening suicide attempts and PID-5 scales}

When the effect of gender was controlled for in nonparametric two-way ANOVAs, a significant association between adolescents' history of life-threatening suicide attempts and PID-5 Submissiveness, $F(1,81)=9.51, p<.01, \eta^{2}=.105$, Anhedonia, $F(1,81)=5.81, p<.05, \eta^{2}=.067$, Depressivity, $F(1,81)=5.02, p<.05, \eta^{2}=.058$, and Unusual beliefs, $F(1$, $81)=6.30, p<.05, \eta^{2}=.072$ trait scales was still observed; no significant effect was observed for PID-5 Withdrawal, $\eta^{2}=$ $.031, p>.10$ trait scale score. When the effect of adolescents' gender was controlled for in nonparametric two-way ANOVA, PID-5 Detachment remained significantly associated with adolescents' history of life-threatening suicide attempts, $F(1$, 81) $=3.99, p<.05, \eta^{2}=.047$. 
Table 3

Participants with history of life-threatening suicidal attempts $(\mathrm{n}=24)$ vs. participants without history of life-threatening suicidal attempts $(\mathrm{n}=61)$ : nonparametric comparisons on Personality Inventory for DSM-5 Trait and Domain Scale Summary Table.

\begin{tabular}{|c|c|c|c|c|c|}
\hline \multirow[b]{2}{*}{ PID-5 Scales } & \multicolumn{2}{|c|}{ HLTSA + $(n=24)$} & \multicolumn{2}{|c|}{ HLTSA - $(n=61)$} & \multirow[b]{2}{*}{$r_{r-b}$} \\
\hline & $M$ & $S D$ & $M$ & $S D$ & \\
\hline Anxiousness & 2.12 & 0.71 & 1.80 & 0.67 & $.31^{*}$ \\
\hline Emotional lability & 2.22 & 0.71 & 2.01 & 0.73 & .20 \\
\hline Hostility & 1.73 & 0.75 & 1.49 & 0.69 & .19 \\
\hline Perseveration & 1.54 & 0.51 & 1.35 & 0.59 & .23 \\
\hline Restricted affectivity & 1.27 & 0.60 & 1.05 & 0.64 & .23 \\
\hline Separation insecurity & 1.78 & 0.84 & 1.47 & 0.69 & .26 \\
\hline Submissiveness & 1.68 & 0.76 & 1.02 & 0.90 & $.45^{* *}$ \\
\hline Anhedonia & 1.90 & 0.69 & 1.40 & 0.69 & $.44 * *$ \\
\hline Depressivity & 2.14 & 0.83 & 1.59 & 0.87 & $.40 * *$ \\
\hline Intimacy avoidance & 1.17 & 0.98 & 0.89 & 0.73 & .14 \\
\hline Suspiciousness & 1.73 & 0.55 & 1.62 & 0.59 & .12 \\
\hline Withdrawal & 1.43 & 0.83 & 1.04 & 0.70 & $.31 *$ \\
\hline Attention seeking & 1.09 & 0.82 & 0.95 & 0.73 & .11 \\
\hline Callousness & 0.56 & 0.54 & 0.56 & 0.49 & .04 \\
\hline Deceitfulness & 0.73 & 0.44 & 0.75 & 0.59 & .07 \\
\hline Grandiosity & 0.33 & 0.50 & 0.50 & 0.54 & .25 \\
\hline Manipulativeness & 0.66 & 0.64 & 0.65 & 0.65 & .03 \\
\hline Distractibility & 1.81 & 0.79 & 1.57 & 0.70 & .22 \\
\hline Impulsivity & 1.88 & 0.80 & 1.51 & 0.89 & .25 \\
\hline Rigid perfectionism & 1.24 & 0.73 & 1.17 & 0.65 & .08 \\
\hline Risk taking & 1.64 & 0.58 & 1.62 & 0.72 & .05 \\
\hline Irresponsibility & 1.09 & 0.64 & 1.11 & 0.60 & .02 \\
\hline Eccentricity & 1.44 & 0.84 & 1.60 & 0.81 & .12 \\
\hline Perceptual dysreg. & 1.16 & 0.74 & 1.20 & 0.69 & .05 \\
\hline Unusual beliefs & 0.65 & 0.66 & 1.13 & 0.74 & $-.41^{* *}$ \\
\hline \multicolumn{6}{|l|}{ PID-5 Domain scales } \\
\hline Negative affectivity & 2.04 & 0.60 & 1.76 & 0.57 & .28 \\
\hline Detachment & 1.50 & 0.74 & 1.11 & 0.59 & $.33 *$ \\
\hline Antagonism & 0.57 & 0.45 & 0.63 & 0.45 & .12 \\
\hline Disinhibition & 1.59 & 0.55 & 1.40 & 0.58 & .24 \\
\hline Psychoticism & 1.08 & 0.66 & 1.31 & 0.67 & .21 \\
\hline
\end{tabular}

HLTSA +: Presence of history of life-threatening suicidal attempts; HLTSA -: Absence of history of life-threatening suicidal attempts; PID-5: Personality Inventory for DSM-5; $r_{r-b}$ : rank biserial $r$ coefficient. Statistical significance for $r_{r-b}$ coefficients was based on Mann-Whitney $U$-tests.

$* p<.05$.

$* * p<.01$.

Nonparametric comparisons between adolescent inpatients with history of life-threatening suicide attempts and adolescent inpatients with no history of life-threatening suicide attempts on PID-5 trait and domain scores are summarized in Table 3.

When the effect of clinical diagnosis of mood disorder was controlled for in nonparametric two-way ANOVAs, a significant association between adolescents' history of life-threatening suicide attempts and PID-5 Depressivity, $F(1$, $81)=4.87, p<.05, \eta^{2}=.057$, Anhedonia, $F(1,81)=5.46$, $p<.05, \eta^{2}=.063$, and Submissiveness, $F(1,81)=5.44$, $p<.05, \eta^{2}=.063$, trait scales was still observed. Rather, no significant association between PID-5 Anxiousness, $\eta^{2}=$ $.023, p>.10$, Withdrawal, $\eta^{2}=.038, p>.05$, and Unusual beliefs, $\eta^{2}=.046, p>.05$, trait scales was observed after controlling for the effect of participants' diagnosis of mood disorders in nonparametric two-way ANOVAs. When the effect of clinical diagnosis of mood disorder was controlled for in nonparametric two-way ANOVA, PID-5 Detachment domain score was not significantly associated with adolescents' history of life-threatening suicide attempts, $\eta^{2}=.033$, $p>.05$.

Finally, after adjusting PID-5 scale scores for the effect of dimensionally-assessed $D S M$-5 Section II BPD, submissiveness $(+), U=427.50, z=2.90, p<.01, r_{r-b}=.42$, anhedonia $(+), U=459.50, z=2.48, p<.01, r_{r-b}=.37$, and unusual beliefs $(-), U=379.50, z=-3.37, p<.001$, $r_{r-b}=-.48$, remained significantly associated with history of life-threatening suicide attempts, whereas the relationship between history of life-threatening suicide attempts and anxiousness, $U=544.50, z=1.74, p>.05, r_{r-b}=.26$, depressivity, $U=543.50, z=1.75, p>.05, r_{r-b}=.26$, and withdrawal, $U=527.00, z=1.91, p<.01, r_{r-b}=.28$, dropped to non-significance. However, the association between PID-5 Detachment domain scale and history of life-threatening suicide attempts remained significant even when PID-5 Detachment scores were adjusted for the effect of dimensionally assessed BPD, $U=522.00, z=1.96$, 
$p<.05, r_{r-b}=.29$. Finally, when we controlled the association between dimensionally assessed BPD and history of life-threatening suicide attempts for the effect of PID-5 Depressivity scale scores, it remained significant, $U=$ 518.50, $z=1.99, p<.05$, but the corresponding $r_{r-b}$ value dropped to .29 .

\section{Discussion}

To our knowledge, this study represents the first attempt at providing data on the reliability and clinical usefulness of the PID-5 in a sample of adolescent inpatients. Although they should be considered only as preliminary evidence, as a whole our data suggested that PID-5 scales may capture reliable variation in maladaptive personality traits and domains among clinically referred adolescents, showing meaningful associations with PD symptoms that were assessed using semi-structured interviews and with adolescents' history of life-threatening suicide attempts.

Confirming and extending De Clercq and colleagues' [5] data on non-clinically referred adolescents, our data suggested that the PID-5 may represent a reliable and useful instrument to assess dysfunctional personality traits in clinically referred adolescents, at least in its Italian translation. Consistent with both adolescent data [5] and adult data (for a review, see [17]), in our study the PID-5 personality trait scales showed adequate internal consistency reliability estimates, with the partial exception of Suspiciousness scale. Consistent with De Clercq and colleagues' [5] findings, in our study Cronbach's $\alpha$ value lower than .70 was observed for PID-5 Suspiciousness scale; although this $\alpha$ coefficient value is usually proposed as a guideline for acceptable reliability, it should be stressed that in our study MIC value suggested adequate internal consistency also for PID-5 Suspiciousness scale [41]. The differences between male adolescents and female adolescents that were observed in our study were partially consistent with adult data on the Italian translation of the PID-5 [23], with and female adolescents showing higher scores than male adolescents on selected internalizing traits (mostly on anxiousness, separation insecurity, and depressivity), as well as on PID-5 Cognitive dysregulation scale.

\subsection{Relationships between dimensionally assessed SCID-II $P D$ diagnoses and PID-5 scales}

Notwithstanding their preliminary nature, our data seemed to yield at least partial support to the construct validity of the PID-5 scales also in clinically referred adolescents, at least in terms of pattern of associations with dimensionally assessed DSM-5 Section II PDs that were also included in the DSM-5 AMPD. Of course, because our participants were all of minor age we were not able to test the expected associations between PID-5 traits and Antisocial PD. Although we relied on different methods in order to assess DSM-5 AMPD traits and DSM-5 Section II PDs, all significant correlations were at least of moderate size.
In our study, all PID-5 traits that were expected to characterize BPD - i.e., anxiousness, emotional lability, hostility, separation insecurity, depressivity, impulsivity, and risk taking - showed significant associations with SCID-II BPD symptoms. This finding was consistent with well-established data on the relationships between PID-5 trait scales and measures of PD symptoms in adults (for a review, see [17]). In our study, consistent with previous studies in adult clinical samples [42,43], and undergraduate student samples $[44,45]$ the PID-5 profile associated with dimensionally assessed SCID-II BPD also included Suspiciousness and Perceptual and Cognitive Dysregulation scales. It is not possible to say whether this latter result is attributable to the subjects' age or more specific psychopathological issues. However, it should be noted that PID-5 Perceptual and Cognitive Dysregulation scales list several items assessing dissociative experiences; thus, this finding seemed to be consistent with the disposition of BPD subjects to experience paranoid ideation or dissociative symptoms [28]. Similarly, a priori DSM-5 trait profiles for Avoidant PD and Narcissistic $\mathrm{PD}$, respectively, were also largely replicated in our sample of Italian adolescent inpatients.

Rather, the PID-5 profile that was observed in our study for Obsessive-Compulsive PD was partially consistent with the DSM-5 a priori trait profile for Obsessive-Compulsive PD. In our adolescent inpatient sample ObsessiveCompulsive PD was significantly associated with PID-5 Rigid perfectionism scale, with no significant contribution of Perseveration and Intimacy avoidance scales; rather, Obsessive-Compulsive PD was characterized by low scores on depressivity, impulsivity, risk taking, irresponsibility, perceptual and cognitive dysregulation, and unusual beliefs. In our opinion, further studies on clinically referred adolescents are needed in order to evaluate if this finding reflects simply sampling issues - indeed, our sample included a non-negligible proportion of adolescents suffering from other specified eating disorders - or if it represents a developmentally appropriate representation of ObsessiveCompulsive PD in adolescence. Finally, the most striking differences between a priori $D S M-5$ trait profile and empirically assessed PID-5 trait profile was observed for Schizotypal PD. Surprisingly, none of the PID-5 scales measuring psychoticism domain traits were significantly associated with dimensionally assessed SCID-II Schizotypal PD, although adolescent inpatients scoring high on PID-5 Withdrawal and Suspiciousness scales received a high number of observer-rated Schizotypal PD symptoms according to SCID-II interview. Although our data were partially consistent with the DSM-5 AMPD trait profile for Schizotypal PD, we think that some of the characteristics of our sample may have biased our results. Indeed, in our sample only six adolescents showed five or more Schizotypal PD criteria; moreover, none of our adolescent inpatients received a diagnosis of psychotic disorder and could be considered at risk for schizophrenia based on family history. Thus, in our study Schizotypal PD might show weak 
associations with Psychoticism scales because none of the adolescent has marked Schizotypal PD qualities. Further studies based on samples including an adequate base rate of Schizotypal PD participants should be carried before accepting our findings on the relationships between PID-5 trait scales and Schizotypal PD.

\subsection{Relationships between history of life-threatening suicide attempts and PID-5 scales}

Our data suggested that the clinical usefulness of PID-5 in adolescent inpatients may extend beyond PDs to profiling adolescents at risk for life-threatening suicide attempts. In our adolescent sample, selected PID-5 trait scales were significantly associated with adolescents' history of life-threatening suicide attempts even after controlling for a number of confounders, including mood disorder diagnosis and dimensionally assessed DSM-5 Section II BPD. As a whole, self-reports on DSM-5 AMPD Anxiousness (+), Submissiveness $(+)$, Anhedonia $(+)$, Depressivity $(+)$, Withdrawal $(+)$, and Unusual beliefs $(-)$ traits were associated with heightened risk for life-threatening suicide in adolescence, at least when we operationalized it as evidence of at least one life-threatening suicide attempt. In particular, adolescent's deficits in the capacity to feel pleasure or take interest in things [i.e., anhedonia] and adaptation of adolescent's behavior to the actual or perceived interests and desires of others [i.e., submissiveness]) were significantly associated with adolescent's history of life-threatening suicide attempts over and above the presence of mood disorder diagnosis and number of DSM-5 Section II BPD features.

The negative association between history of life-threatening suicide attempts and unusual beliefs was somewhat unexpected. In our opinion, it seems to stress that non-psychotic clinically referred adolescents with prominent maladaptive personality features may see life-threatening suicide attempts as a rational ways to cope with life difficulties and dispositional hopelessness rather than as results of dysfunctional cognitive appraisal of life events. However, it is also possible that being "stuck in reality" with very limited possibilities to fly into fantasy - or to dissociate from overwhelming emotional states - in response to life difficulties (i.e., low Unusual beliefs) may represent a trigger for severe suicide behavior in non-psychotic clinically referred adolescents who are characterized by a disposition toward anxiety, depressive feelings, hopelessness, lack of interests/ pleasure (i.e., anhedonia), and poor assertiveness (i.e., submissiveness). In other terms, according to our data severe emerging personality pathology may be associated with life-threatening suicide attempts in clinically referred adolescents over and above mood disorders. In particular, clinically referred adolescents prone to experience anxiety, depressive states, hopelessness, who see themselves as poorly assertive and with lack of enjoyment from, or energy for life's experiences, and are characterized by an excessively rational and concrete approach to reality may consider suicide as the only viable solution when they feel stuck in a life situation that they feel unable to cope with or change to any extent, but who they feel as dramatically real and inescapable.

Interestingly, in our study the association between life-threatening suicide attempts in adolescence and adolescent's reports of feelings of being down, miserable, and/or hopeless (i.e., depressivity) was selectively mediated by the number of DSM-5 Section II BPD features that were met by each adolescent. This finding, as well as the substantial relationship between BPD and Depressivity that was observed in our sample, are consistent with previous reports suggesting a central role of hopelessness/helplessness feelings in the clinical management of BPD [e.g.,46-48]. In our opinion, our findings are consistent with a consistent body of research showing that that the assessment of DSM-5 AMPD traits may provide clinically relevant information both on personality pathology and on a number of constructs that extend beyond PDs [17]. Thus, our data suggest the clinicians considering to include PID-5 in the routine assessment of adolescent inpatients; at least, clinicians should monitor the presence of indicators of anhedonia submissiveness when estimating the risk for life-threatening suicide attempts in adolescent inpatients.

\subsection{Limitations}

Of course, our findings should be considered in the light of several limitations. Our sample was composed only of adolescent inpatients who were characterized by a high prevalence of DSM-5 Cluster B PDs, and by a reduced range of psychiatric disorder diagnoses; this inherently limits the generalizability of our results to other clinical samples, and suggest that Berkson's bias should also be kept in mind when studying clinical samples. We relied on a cross-sectional design; this inherently prevented us from studying causal relationships between adolescents' history of life-threatening suicide attempts and PID-5 variables. The sample size was definitively too small to legitimate the use of sophisticated multivariate analyses in order to evaluate the predictive role of PID-5 scales on SCID-II data and adolescents' history of life-threatening suicide attempts. Moreover, the majority (77.6\%) of participants included in the present sample were female, and this inherently limits the generalizability of our findings to samples composed of male adolescents.

We were able to obtain only dichotomously assessed (i.e., presence/absence) history of life-threatening suicidal attempts from clinicians who were following in treatment our adolescent inpatients; unfortunately we were not able to obtain reliable data on the frequency of life-threatening suicidal attempts, as well as on frequency and severity of non-suicidal self-injurious behavior. Another important limitation to the present study is that DSM-IV Axis I diagnoses were not assessed using standardized interviews. We were not able to assess inter-rater reliability of dimensional SCID-II PD diagnoses and categorical DSM-IV axis I clinical diagnoses/ presence of history of life-threatening suicide attempts. This 
may have somewhat biased our findings, although usually poor reliability leads to attenuation of association, thus placing our findings on the conservative side.

We relied only on a single interview to evaluate $D S M-5$ Section II PD symptoms; since available data suggest that $D S M$-based measures of PDs may show poor convergent validity [31], our findings should not be uncritically generalized to patients that were assessed using other interviews for PD diagnosis.

\section{Conclusions}

Even keeping these limitations in mind, we think that our findings may provide useful, albeit preliminary data on the clinical usefulness of PID-5 in the assessment of adolescent inpatients.

\section{Conflicts of interest}

On behalf of all authors, the corresponding author states that there is no conflict of interest.

\section{References}

[1] Sharp C, Bleiberg E. Borderline personality disorder in children and adolescents. In: Martin A, \& Volkmar F, editors. Lewis' child and adolescent psychiatry: comprehensive textbook. Baltimore: Lippincott Williams and Wilkins; 2007. p. 680-91.

[2] Irwin CE. Risk taking behaviors in the adolescent patient: are they impulsive? Pediatr Ann 1989;18:122-33.

[3] Irwin CE, Burg SJ, Uhlercart C. America's adolescents: where have we been, where are we going? J Adolesc Health 2002;31:91-121.

[4] Bleiberg E, Rossouw T, Fonagy P. Adolescent breakdown and emerging borderline personality disorder. In: Bateman AW, \& Fonagy P, editors. Handbook of metalizing in mental health practice. Arlington: American Psychiatric Publishing; 2011. p. 463-509.

[5] De Clercq B, De Fruyt F, De Bolle M, Van Hiel A, et al. The hierarchical structure and construct validity of the PID-5 trait measure in adolescence. J Pers 2014;82:158-69.

[6] De Fruyt F, De Clercq B. Antecedents of personality disorder in childhood and adolescence: toward an integrative developmental model. Annu Rev Clin Psychol 2014;10:449-76.

[7] Chanen AM. Outcomes in women diagnosed with borderline personality disorder in adolescence: early detection and timely intervention are fundamental. J Can Acad Child Adolesc Psychiatry 2011;20:175.

[8] Sharp C, Williams LL, Ha C, Baumgardner J, Michonski J, et al. The development of a mentalization-based outcomes and research protocol for an adolescent inpatient unit. Bull Menn Clin 2009;73:311-38.

[9] Michonski JD, Sharp C, Steinberg L, Zanarini MC. An item response theory analysis of the DSM-IV borderline personality disorder criteria in a population-based sample of 11- to 12-year-old children. Pers Disord 2013;4:15-22.

[10] Miller AL, Muehlenkamp JJ, Jacobson CM. Fact or fiction: diagnosing borderline personality disorder in adolescents. Clin Psychol Rev 2008;28:969-81

[11] Sharp C, Ha C, Michonski J, Venta A, Carbone C. Borderline personality disorder in adolescents: evidence in support of the childhood interview for $D S M-I V$ borderline personality disorder in a sample of adolescent inpatients. Compr Psychiatry 2012;53:765-74.

[12] Tackett JL, et al. A unifying perspective on personality pathology across the life span: developmental considerations for the fifth edition of the diagnostic and statistical manual of mental disorders. Dev Psychopathol 2009;21:687-713.

[13] Helzer JE, Kraemer HC, Krueger RF. The feasibility and need for dimensional psychiatric diagnoses. Psychol Med 2006;36:1671-80.

[14] American Psychiatric Association. Diagnostic and statistical manual of mental disorders. 3rd ed. Washington: Author; 1980.

[15] Widiger TA, Trull TJ. Plate tectonics in the classification of personality disorder. Am Psychol 2007;62:71-83.

[16] American Psychiatric Association. Diagnostic and statistical manual of mental disorders. 4th ed. Washington: Author; 1994.

[17] Krueger RF, Markon KE. The role of the DSM-5 personality trait model in moving toward a quantitative and empirically based approach to classifying personality and psychopathology. Annu Rev Clin Psychol 2014;10:477-501.

[18] Clark LA. Assessment and diagnosis of personality disorder: perennial issues and emerging conceptualization. Annu Rev Clin Psychol 2007;58:227-58.

[19] Harkness AR, McNulty JL, Ben-Porath YS. The personality psychopathology five (PSY-5): constructs and MMPI-2 scales. Psychol Assess 1995;7:104-14.

[20] Trull TJ, Durrett CA. Categorical and dimensional models of personality disorder. Annu Rev Clin Psychol 2005;1:355-80.

[21] Widiger TA, Simonsen E. Alternative dimensional models of personality disorder: finding a common ground. J Personal Disord 2005;19:110-30.

[22] Krueger RF, Derringer J, Markon KE, Watson D, Skodol AE. Initial construction of a maladaptive personality trait model and inventory for DSM-5. Psychol Med 2012;42:1879-90.

[23] Fossati A, Krueger RF, Markon KE, Borroni S, Maffei C. Reliability and validity of the personality inventory for $D S M-5$ (PID-5): predicting DSM-IV personality disorders and psychopathy in communitydwelling Italian adults. Assessment 2013;20:689-708.

[24] Glenn CR, Bagge CL, Osman A. Unique associations between borderline personality disorder features and suicide ideation and attempts in adolescents. J Pers Disord 2013;27:604.

[25] Muehlenkamp JL, et al. International prevalence of adolescent nonsuicidal self-injury and deliberate self-harm. Child Adolesc Psychiatr Ment Health 2012;6:1-9.

[26] Homan KJ, LA S, JD F, MP T. Five-year prospective investigation of self-harm/suicide-related behaviors in the development of borderline personality disorder. Pers Disord 2016.

[27] MB F, RL S, Gibbon M, Williams JBW, Benjamin L. Structured clinical interview for DSM-IV Axis II personality disorders (SCID II) version 2.0. New York: Biometrics Research Department, NY State Psychiatric Institute; 1994.

[28] American Psychiatric Association. Diagnostic and statistical manual of mental disorders. 5th ed. Washington: Author; 2013.

[29] Denissen JJ, Geenen R, van Aken MA, Gosling SD, Potter J. Development and validation of a Dutch translation of the big five inventory (BFI). J Pers Assess 2008;90:152-7.

[30] Maffei C, Fossati A, Agostoni I, Barraco A, et al. Interrater reliability and internal consistency of the structured clinical interview for DSM$I V$ axis II personality disorders (SCID-II) version 2.0. J Personal Disord 1997;11:279-84.

[31] Zimmerman M. Diagnosing personality disorders. A review of issues and research methods. Arch Gen Psychiatry 1994;51:225-45.

[32] American Psychiatric Association. Online assessment measures. The personality inventory for DSM-5 (PID-5) - child age 11-17; 2013 [Retrieved from, http://www.psychiatry.org/practice/dsm/dsm5/onlineassessment-measures\#Personality].

[33] Anderson JL, Sellbom M. Construct validity of the DSM-5 section III personality trait profile for borderline personality disorder. J Pers Assess 2015;97:478-86. 
[34] Richman H, Nelson-Gray R. Nonclinical panicker personality: profile and discriminative ability. J Anxiety Disord 1994;8:33-47.

[35] Cureton EE. Rank-biserial correlation. Psychometrika 1956;21:287-90.

[36] Wobbrock JO, Findlater L, Gergle D, Higgins JJ. The aligned rank transform for nonparametric factorial analyses using only ANOVA procedures. Proceedings of the SIGCHI conference on human factors in computing systems; 2011. p. 143-6.

[37] Fawcett RF, Salter KC. A Monte Carlo study of the F test and three tests based on ranks of treatment effects in randomized block designs. Commun Stat Simul Comput 1984;13:213-25.

[38] Salter KC, Fawcett RF. A robust and powerful rank test of treatment effects in balanced incomplete block designs. Commun Stat Simul Comput 1985;14:807-28.

[39] Hodges JL, Lehmann EL. Rank methods for combination of independent experiments in the analysis of variance. Ann Math Stat 1962;33:482-97.

[40] R Core Team. R: a language and environment for statistical computing. Vienna: R Foundation for Statistical Computing; 2015.

[41] Clark LA, Watson D. Constructing validity: basic issues in objective scale development. Psychol Assess 1995;7:309-19.
[42] Bach B, Anderson J, Simonsen E. Continuity between interview-rated personality disorders and self-reported DSM-5 traits in a Danish psychiatric sample. Pers Disord 2016, http://dx.doi.org/10.1037/ per0000171.

[43] Sellbom M, Sansone RA, Songer DA, Anderson JL. Convergence between DSM-5 section II and section III diagnostic criteria for borderline personality disorder. Psychiatry 2014;48:325-32.

[44] Anderson J, Snider S, Sellbom M, Krueger R, Hopwood C. A comparison of the DSM- 5 section II and section III personality disorder structures. Psychiatry Res 2014;216:363-72.

[45] Hopwood CJ, Thomas KM, Markon KE, Wright AG, Krueger RF. DSM-5 personality traits and DSM-IV personality disorders. J Abnorm Psychol 2012;121:424-32.

[46] Gunderson JG, Phillips KA. A current view of the interface between borderline personality disorder and depression. Am J Psychiatry 1991;148:967-75.

[47] Masterson J. The search for the real self. New York: The Free Press; 1988.

[48] Trull T. Structural relations between borderline personality disorder features and putative etiological correlates. J Abnorm Psychol 2001;110:471-81. 